Supplementary Information for

\title{
Peptide-based Biosensing of Redox-active Protein-Heme Complexes Indicates Novel Mechanism for Tumor Survival under Oxidative Stress
}

Kai Zhang, *, a Zhenqiang Fan, ${ }^{a, c}$ Jiena Weng, ${ }^{c}$ Jianfeng Zhao, ${ }^{c}$ Jiaying Wang, ${ }^{d}$ Hao Wu, ${ }^{a}$ Minhao Xie, ${ }^{a}$ Hong Zhou, ${ }^{*}, e$ Hao $\mathrm{Li}^{*}, b$

${ }^{a}$ Key Laboratory of Nuclear Medicine, Ministry of Health, Jiangsu Key Laboratory of Molecular Nuclear Medicine, Jiangsu Institute of Nuclear Medicine. Wuxi, Jiangsu 214063, China.

${ }^{b}$ School of Biological Science and Technology, University of Jinan, No. 106 Jiwei Road, Jinan, Shandong 250022, China

${ }^{c}$ Key Laboratory of Flexible Electronics (KLOFE) \& Institute of Advanced Materials (IAM), Jiangsu National Synergetic Innovation Center for Advanced Materials (SICAM), Nanjing Tech University (NanjingTech), 30 South Puzhu Road, Nanjing 211816, P.R.

China

${ }^{d}$ Department of Rehabilitation \& Acupuncture and Moxibustion, Nanjing Medical University, Affiliated Wuxi People’s Hospital. Wuxi, Jiangsu 214000, China.

${ }^{e}$ Key Laboratory of Optic-electric Sensing and Analytical Chemistry for Life Science, Ministry of Education; Shandong Key Laboratory of Biochemical Analysis; Key Laboratory of Analytical Chemistry for Life Science in Universities of Shandong; College of Chemistry and Molecular Engineering, Qingdao University of Science and Technology, Qingdao 266042, P. R. China

\footnotetext{
* Corresponding author. Fax: +86-510-85508775; Tel: +86-510-85508775

E-mail addresses: cardely@126.com (H. Li), zhangkai@jsinm.org (K. Zhang), zhouhong@qust.edu.cn (H, Zhou)
} 

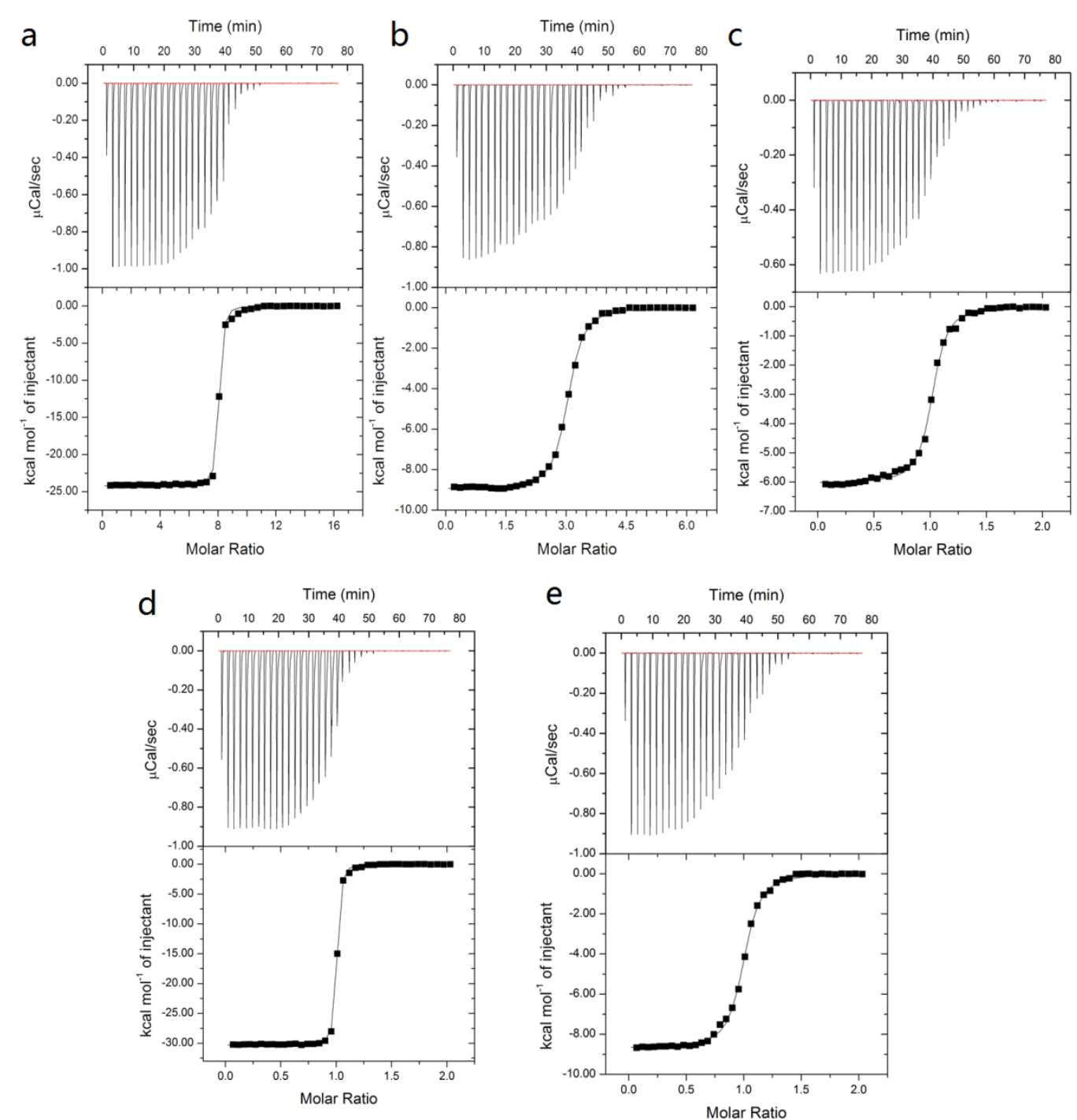

Figure S1. Isothermal titration calorimetry (ITC) titration of (a) $0.5 \mathrm{mM}$ ferritin peptide probe into $0.00625 \mathrm{mM}$ ferritin, (b) $1 \mathrm{mM}$ heme into $0.033 \mathrm{mM}$ ferritin, (c) $500 \mu \mathrm{M}$ p53 into $50 \mu \mathrm{M}$ ferritin, (d) $0.5 \mathrm{mM}$ p53 peptide probe into $0.05 \mathrm{mM} \mathrm{p} 53$, (e) $1 \mathrm{mM}$ heme into $0.1 \mathrm{mM}$ p53. The solution adopted is $10 \mathrm{mM}$ PBS, $\mathrm{pH}$ 7.4. In each panel, the top row displays the raw data of power versus time. The bottom row is the corresponding data by integrating enthalpy values versus the molar ratio of titrant: titrand. These data are fit using Origin 7.0 software, the resulted fitting curve is also shown in the lower row. The obtained affinity (dissociation constant) is (a) $3.6 \times 10^{-7} \mathrm{M}$ for one site, (b) $5.9 \times 10^{-6} \mathrm{M}$ for one site, (c) $1.2 \times 10^{-6} \mathrm{M}$, (d) $1.45 \times 10^{-8} \mathrm{M}$, (d) $2.3 \times 10^{-6}$ $\mathrm{M}$ 


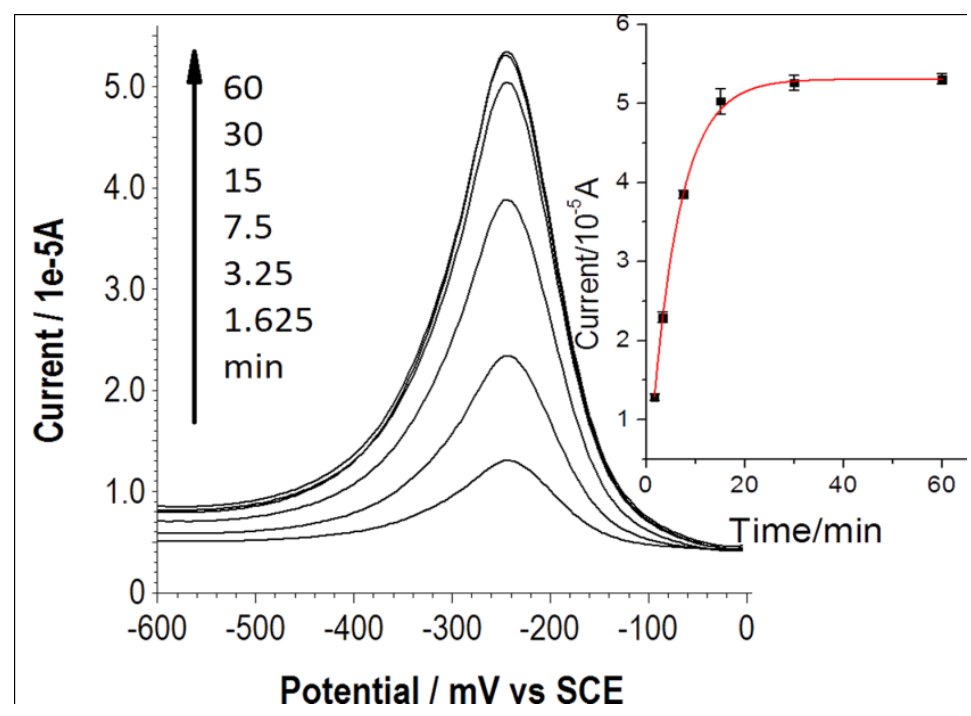

Figure S2. Optimization of the incubation time for ferritin-probe peptide interaction, using the electrochemically released Fe(II) ion as the signal readout. Inset is the kinetics of binding recorded by plotting the peak currents of $\mathrm{Fe}$ (II) as a function of incubation time (Error bars represent standard deviation of repetitive measurments).

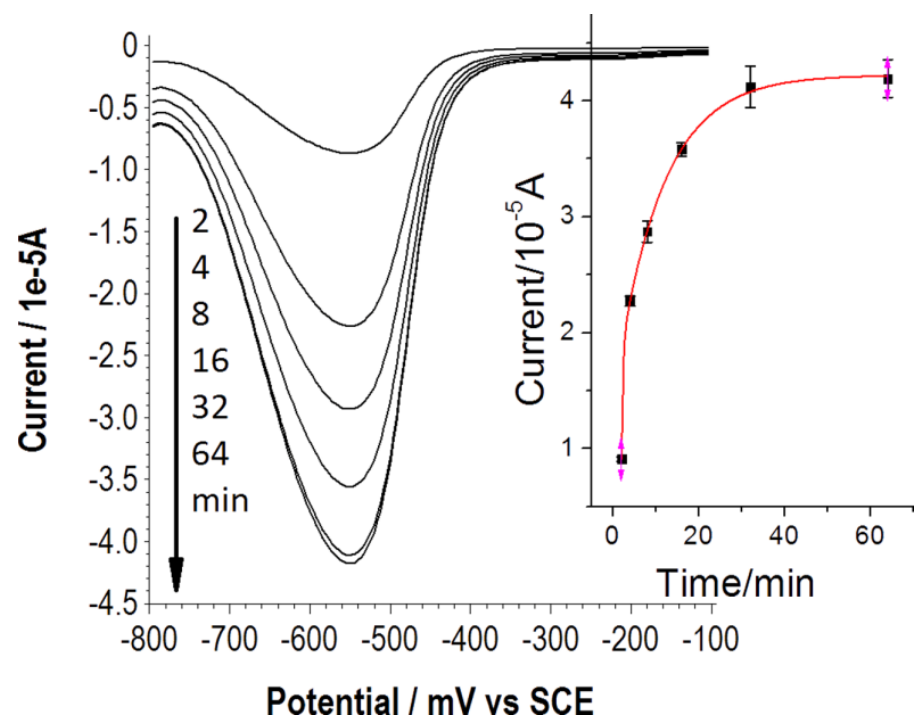

Figure S3. Similar optimization of incubation time for p53-probe peptide interactions. 

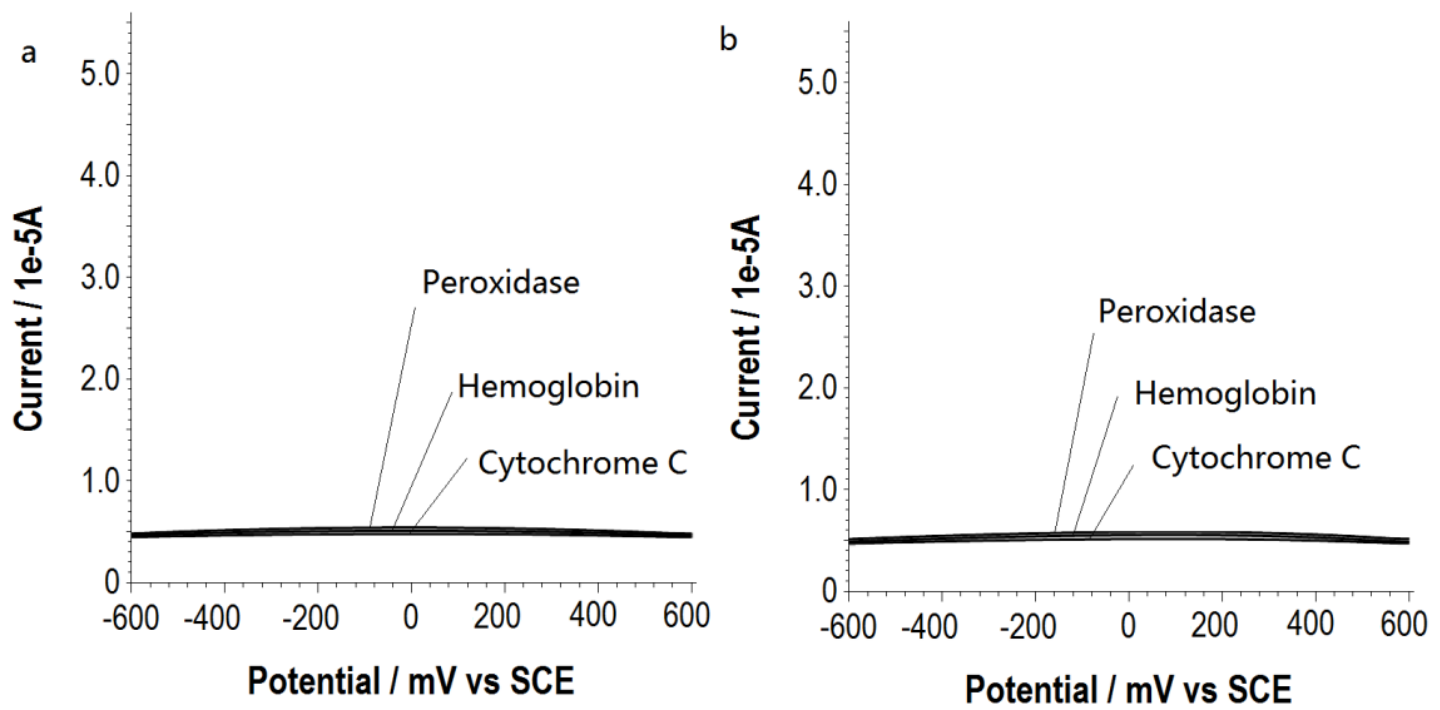

Figure S4. Control experiments testing the specificity of the ferritin probe or probe 1 (a), and the specificity of the p53 probe or probe 2 (b). Both probes have been incubated with control heme proteins as shown, and the electrochemical response of these heme proteins have been recorded. It can be seen that the recorded response is negligible, indicating almost no heme protein been captured on the probes. 